was $90.1 \%$ on immediate angiography and was $96.4 \%$ at the last follow-up. Clinical outcomes and follow-up aneurysm occlusion did not significantly differ between within 24 hours and 25-72hours groups.

Conclusions Our results showed that the timing of treatment was an independent predictor of perioperative complications after stent-assisted coiling of acutely ruptured aneurysms. The LVIS stent-assisted coiling may be safe and effective in the treatment of acutely ruptured intracranial aneurysms within 24 hours with low perioperative complication rates.

Disclosures B. Zhao: None. X. Wang: None. Y. Chen: None. J. Cai: None. D. Jin: None. J. Wan: None. Y. Pan: None. G. Mao: None.

\section{E-192 BEYOND SIDE WALL LESIONS: FLOW DIVERTER EXPERIENCE TO TREAT MCA BIFURCATION ANEURYSMS}

B Pabon Guerrero*, M Patino Hoyos, J Gutierrez Banos, V Torres, J Mejia. Neurointevencionismo, AngiTeam, Medellin, Colombia

\subsection{6/neurintsurg-2020-SNIS.223}

Background Flow Diverter Devices (FDD) have been shown to be effective in the treatment of side-wall, wide-necked IA. However, the use of these devices to treat bifurcation lesions remains to be validated. MCA wide-neck IA. Represents a frequent finding in our daily practice and constitutes a real challenge for endovascular conventional approach requiring special considerations: (Balloon Assisted Coiling, Stent and Coils). Few studies have been conducted on the safety and efficacy using FDD for the treatment of MCA bifurcation aneurysms. Objective To describe our experience using FDD in the treatment of MCA IA. Feasibility, Safety and Efficacy are reported. Radiological (Complete Occlusion rate) and, Clinical findings were registered immediately after implantation and during follow-up. Factors associated with incomplete occlusion were analyzed.

Methods We retrospectively analyzed a subgroup of patients with MCA IA treated using FDD in our data-base. We describe here clinical, anatomical and angiographic variables. Procedural technical success, adverse events and Follow Up were registered. Complete Occlusion rate was defined using O'Kelly-Marotta classification.

Results From June 2012 to April 2020 a total of 62 patients with 64 aneurysms were selected in this series. All cases under DAPT and GA. Giant: 11, Large: 9 and Small lesions: 44 $(68 \%)$. Fourteen cases with history of SAH. A total of 71 devices were implanted (PED Classic :6, PED Flex: 21, PED Flex-shield:18 and FRED: 26). A mean of 1.1 device per aneurysm. In all cases planned but two (PED Classic) FD finally could be implanted i.e., Technical success of $97 \%$. Radiological FU available in 38/64 (60\%). Mid-long term Follow-up (mean: 29 months). showed a complete occlusion rate of $78 \%$. MCA major branch with flow reduction or acute occlusion was evidenced in 13 cases (20\%). Of these, six cases were solved using IIb-IIIa GPI and seven cases (10.9\%) remain occluded and evolved with neurological clinical deficit.

Conclusion The use of FD's technology for treatment of MCA bifurcations IA should be reserved for highly selected patients otherwise considered un-treatable with conventional endovascular approaches. Learning curve, accurate DAPT and intraprocedural arterial blood pressure supervision must be considered as key factors to avoid undesired thrombo-embolic complications. The real role of FD's and its comparison with endosaccular approaches remain to be defined in posterior and controlled studies.

Disclosures B. Pabon Guerrero: None. M. Patino Hoyos: None. J. Gutierrez Banos: None. V. Torres: None. J. Mejia: None.

\section{E-193 CELLULAR RESPONSES TO FLOW DIVERTERS IN A TISSUE-ENGINEERED ANEURYSM}

${ }^{1} \mathrm{~W}$ Liu, ${ }^{1} \mathrm{D}$ Ding, ${ }^{1} \mathrm{Y}$ Ding, ${ }^{2} \mathrm{~K}$ Temnyk, ${ }^{2} \mathrm{~T}$ Shen, ${ }^{2} \mathrm{~K}$ O'Halloran Cardinal, ${ }^{1} \mathrm{D}$ Kallmes, ${ }^{1} \mathrm{R}$ Kadirvel*. ${ }^{1}$ Radiology, Mayo Clinic, Rochester, MN; ${ }^{2}$ Biomedical Engineering Department, Cal Poly, San Luis Obispo, CA

\subsection{6/neurintsurg-2020-SNIS.224}

Background and Objective Notwithstanding the widespread implementation of flow diverters (FDs) in the treatment of intracranial aneurysms, the exact mechanism of action of these devices remains elusive. The aim of this study is to advance the understanding of cellular responses to FD implantation using a 3D tissue engineered in vitro aneurysm model.

Methods Aneurysm-like blood vessel mimics (aBVMs) were constructed by first electrospinning polycaprolactone nanofibers on desired aneurysm-like geometries to create scaffolds. After conditioning, aBVMs were mounted into sterile bioreactor chambers and incubated with a cell culture media under steady state flow. Then aBVMs were sodded with human carotid smooth muscle cells (SMCs) followed by endothelial cells (ECs). Then, appropriately sized FDs (Pipeline Embolic Device, Medtronic, CA) were deployed in the parent vessel of aBVMs under fluoroscopic guidance, covering the neck. AVBMs with implanted flow diverters were cultivated for 7,14 or 28 days $(n=3$ for each time point). En face immunofluorescence imaging of the aBVMs was performed for EC and SMC markers. The cell coverage in the neck was measured semiquantitatively.

Results The mean diameter of the parent vessel of aBVMs was $4.50 \mathrm{~mm}$. The mean neck diameter, width, and height were 6 $\mathrm{mm}, 5.0 \mathrm{~mm}, 5.0 \mathrm{~mm}$, respectively. The device segment in the parent artery was completely endothelialized at 7 days. The majority of device struts, but not the pores between struts, at the parent artery and the neck interface were partially covered with ECs and SMCs. The device struts in the middle of the neck were lacking cell coverage. At 14 days, histology verified that a neointimal lining like human tissue had formed coverage. Translucent tissue islands partially covering both the struts and pores in the center of the neck were observed. Almost the entire necks of aBVMs were covered with translucent neointimal layer at 28 days. A higher degree of cellular coverage was seen on the struts and pores at the neck at 28 days compared to both 7 and 14 days.

Conclusion Implantation of FDs in the tissue engineered aneurysm model simulates the cellular responses demonstrated in the experimental rabbit model. ABVMs would be a valuable alternative tool for evaluating the healing mechanisms of endovascular aneurysm devices.

Disclosures W. Liu: None. D. Ding: None. Y. Ding: None. K. Temnyk: None. T. Shen: None. K. O'Halloran Cardinal: None. D. Kallmes: None. R. Kadirvel: 1; C; NIH grant\# NS076491. 